\title{
Apical hyphal extension in Streptomyces coelicolor A3(2)
}

\author{
D. I. Gray, G. W. Gooday and J. I. Prosser* \\ Department of Genetics and Microbiology, University of Aberdeen, Marischal College, Aberdeen AB9 IAS, UK
}

(Received 21 August 1989; revised 22 January 1990; accepted 5 February 1990)

\begin{abstract}
Hyphal extension in the filamentous actinomycete Streptomyces coelicolor A3(2) was shown to occur by addition of newly synthesized wall material in an apical extension zone. Incubation of mycelia with tritiated $\boldsymbol{N}$-acetyl-Dglucosamine (GlcNAc), a precursor of peptidoglycan, resulted in localized incorporation of label at the apex, as indicated by light microscopic and electron microscopic autoradiography. Within the hyphal extension zone there was a sharp decrease in incorporation with increasing distance from the apex. Hyphal tip shape, examined by lowtemperature scanning electron microscopy, approximated to a semi-ellipsoid of revolution and was not hemispherical. Tip shape could be represented accurately by polynomial equations of degree less than seven. The surface stress theory was successfully applied to hyphal tip growth, with tip shape related qualitatively to the inverse of surface tension within the wall of the extension zone. Surface tension was assumed to be inversely proportional to the rate of incorporation of tritiated GIcNAc. Treatment of surface-grown hyphae with $\beta$-lactam antibiotics resulted in localized swelling of hyphal tips. Lysozyme caused swelling of tips and of other regions of hyphae, frequently giving a beaded morphology associated with septa.
\end{abstract}

\section{Introduction}

The actinomycetes exhibit a continuous range of growth form from organisms, e.g. coryneforms, which are typically unicellular, to the truly mycelial streptomycetes. Streptomyces coelicolor A3(2) is a typical member of this latter group, forming a mycelium consisting of branching filamentous hyphae whose growth kinetics are similar in many respects to those of filamentous fungi (Allan \& Prosser, 1983, 1985). Individual hyphae extend at a constant linear rate on solid media and the absence of a buckling effect or zig-zag hyphal morphology behind the tip implies that hyphal extension occurs by incorporation of newly synthesized wall material at the hyphal apex. In filamentous fungi, there is strong evidence for tip extension through incorporation of material synthesized in distal regions of the hypha and transported to the tip within membrane-bound vesicles (Gooday \& Trinci, 1980). Streptomycete hyphae have a diameter less than $1 \mu \mathrm{m}$, making detailed study of hyphal growth mechanisms difficult, but Locci \& Schaal (1980) demonstrated apical growth of actinomycete hyphae using immunofluorescent labelling of wall components, and Brana et al. (1982) reported apical incorporation of

\footnotetext{
Abbreviations: GlcNAc, $N$-acetyl-D-glucosamine; LTSEM, lowtemperature scanning electron microscopy; TEM, transmission electron microscope.
}

tritiated $N$-acetyl-D-glucosamine (GlcNAc) by Streptomyces antibioticus.

For hyphae of filamentous fungi, a number of mathematical models have been proposed which relate the mechanism of addition of new wall material to the shape adopted by the hyphal extension zone. Early models (e.g. Green, 1974) assumed the hyphal apex to be hemispherical and growth to be isotropic, and these predicted a cosine relationship between the specific rate of wall expansion and distance from the hyphal apex. Trinci \& Saunders (1977) challenged the assumption of hemispherical tip shape and suggested, on the basis of experimental observations of tip shape, that hyphal tips were approximated better by semi-ellipsoids of revolution. This predicted a cotangent relationship between the specific rate of wall growth and distance from the apex, which fitted qualitatively with data on vesicle concentration, rates of incorporation of tritiated GlcNAc and direct measures of wall expansion. Saunders \& Trinci (1979) proposed that the shape of the hyphal tip resulted from an interaction between the internal hydrostatic pressure, resulting from growth, and the elastic properties of the tip wall, reflecting rates of incorporation of new material. The shape adopted is that which minimizes surface energy within the apical wall. By equating wall elasticity with the specific rate of wall expansion, they obtained predictions of hyphal tip shape which fitted experimental observations well and suggested a 
rapid decrease in wall elasticity with increasing distance from the apex, culminating in complete rigidification at the base of the extension zone, resulting in a constant hyphal diameter.

A similar approach was adopted in the surface stress theory of Koch, which has been used to describe the shape and growth form of several bacteria (see Koch, 1983, 1985) and of filamentous fungi (Koch, 1982). This states that the pressure-volume work resulting from growth is equivalent to the surface tension-area work resulting from wall growth, that is: $P \mathrm{~d} V=T \mathrm{~d} A$, where $P$ represents hydrostatic pressure difference across the tip wall, $\mathrm{d} V$ the incremental increase in volume due to cytoplasmic growth, $T$ the surface tension within the wall and $\mathrm{d} A$ the incremental increase in surface area due to wall growth. By geometric calculation of $\mathrm{d} V$ and $\mathrm{d} A$ during a small interval of time, and assuming $P$ to be constant, Koch (1982) showed that the slope at any point on the tip wall divided by the tip radius at that point is inversely related to $T$. In turn $T$ will be inversely related to the specific rate of wall expansion. Direct measurement of tip shape from micrographs, and comparison with indirect and direct measures of wall expansion, provided strong support for the application of the surface stress theory to fungal hyphal extension.

In this paper we describe further evidence for apical growth of streptomycete hyphae and application of the surface stress theory to apical growth of $S$. coelicolor. In addition, treatment of hyphae with a range of wallsoftening enzymes provides a qualitative test of the theory not previously attempted.

\section{Methods}

Organism and growth media. Streptomyces coelicolor A3(2) was kindly supplied by Professor D. A. Hopwood from the culture collection of the John Innes Institute, Norwich, UK. Spore suspensions were obtained from frozen stocks originally harvested from oatmeal agar and stored in $10 \%(v / v)$ glycerol as described by Allan \& Prosser (1983). Routine cultivation was carried out on minimal medium (MM) (Hopwood, $1960)$ containing Oxoid agar no. $3(1.5 \%, w / v)$ and glucose at a final concentration of $0.4 \mathrm{~g} \mathrm{1}^{-1}$. All growth experiments were carried out in $2 \%(w / v)$ liquid malt extract medium (MEM) or on MM agar.

Light microscopic autoradiography. Incorporation of radioactively labelled $N$-acetyl-D-[1-3 $\mathrm{H}]$ glucosamine (GlcNAc) (Amersham) was investigated using submerged slide cultures. Microscope slides, cleaned in chromic acid and heat-sterilized, were immersed in a subbing solution of gelatin $(5 \%, \mathrm{w} / \mathrm{v} ; \mathrm{BDH})$ and chromium potassium sulphate $(0.5 \%, w / v)$ sterilized by UV irradiation for $2 \mathrm{~d}$. Slides were removed, allowed to air-dry and then placed in Petri dishes and inoculated with $50 \mu \mathrm{l}$ of a spore suspension containing $10^{6}-10^{7}$ spores $\mathrm{ml}^{-1}$. After $5 \mathrm{~min}$, to allow spores to adhere to the treated slides, excess liquid was removed and $10 \mathrm{ml}$ MEM added, followed by incubation at $25^{\circ} \mathrm{C}$ without shaking. In pulse-labelling experiments, tritiated GlcNAc [specific activity $3.4 \mathrm{Ci} \mathrm{mmol}^{-1}\left(125.8 \mathrm{GBq} \mathrm{mmol}^{-1}\right) ; 1 \mu \mathrm{Ci} \mathrm{m} l^{-1}$ in $1 \mathrm{ml}$ MEM] was added to colonized slides which were incubated at $25^{\circ} \mathrm{C}$ for pulse periods of $1,2,3,4,5,10$ and $15 \mathrm{~min}$. In pulse-chase experiments, excess radiolabelled substrate was removed by dipping slides in distilled water, and non-radioactive GlcNAc $[10 \mathrm{mg}(\mathrm{ml}$ $\mathrm{MEM})^{-1}$ ] was applied before further incubation for 0 (control) or $2.5 \mathrm{~min}$. In another series of experiments, the chase was applied for $2.5 \mathrm{~min}$ after a pulse-labelling time of $12 \mathrm{~h}$ commencing immediately after inoculation of slides. A double-labelling experiment was also carried out in which inoculated slides were incubated in tritiated GlcNAc $\left(2 \mu \mathrm{Ci} \mathrm{ml}^{-1}\right)$ for $26 \mathrm{~h}$ after which a second pulse $\left(1 \mu \mathrm{Ci} \mathrm{ml} l^{-1}\right)$ was applied for $165 \mathrm{~min}$. Four control slides were prepared for each labelling experiment by ethanol fixation $(60 \%, \mathrm{v} / \mathrm{v}, 10 \mathrm{~min})$ of two uninoculated slides and two slide cultures ( $12 \mathrm{~h}$ old). One of each type was pulse-labelled for $15 \mathrm{~min}$ with $1 \mu \mathrm{Ci}$ tritiated GlcNAc. All slides were dipped in distilled water and air-dried. Slides were then coated with Kodak AR 10 stripping film as described by Rogers (1973). The quality of the stripping film was monitored by coating two blank subbed slides and exposing one to daylight. All slides were exposed, incubated for $14 \mathrm{~d}$ at $4{ }^{\circ} \mathrm{C}$ and then developed using Kodak D-19 $\left(20^{\circ} \mathrm{C}, 5 \mathrm{~min}\right), 2 \%(\mathrm{v} / \mathrm{v})$ acetic acid $(30 \mathrm{~s})$ and Kodak Unifix ( $\left.5 \mathrm{~min}\right)$, rinsed $(30 \mathrm{~min})$ in water and air-dried. Autoradiograms were mounted in distilled water and observed under bright-field or dark-field microscopy using an Olympus BH-2 microscope interfaced to a CCD camera (Matasushita) and video monitor (Hitachi). The screen image was traced onto acetate overhead projector sheets which were then analysed to determine quantitatively the intensity of labelling as a function of distance from the hyphal apex.

Electron microscopic autoradiography. Samples $(1 \mathrm{ml})$ of overnight cultures of $S$. coelicolor in MEM were centrifuged at $13000 \mathrm{~g}$ for $30 \mathrm{~s}$, resuspended in $1 \mathrm{ml}$ MEM containing $1 \mu \mathrm{Ci}$ tritiated GlcNAc and incubated at $25^{\circ} \mathrm{C}$ for $1,2,3,4$ and $5 \mathrm{~min}$. Samples $(200 \mu 1)$ of treated and unlabelled mycelia were fixed by addition of $200 \mu \mathrm{l}$ absolute ethanol, centrifuged at $13000 \mathrm{~g}$ for $1 \mathrm{~min}$, suspended in $500 \mu \mathrm{l}$ phenol $(2 \%, \mathrm{w} / \mathrm{v})$, heated to $100^{\circ} \mathrm{C}$ for $30 \mathrm{~min}$, centrifuged and resuspended in distilled water. Ilford L. 4 Nuclear Research Emulsion was applied by either the loop technique or the flat substrate technique of Caro \& van Tubergen (1962). Prepared specimens were spread on to Formvarcoated nickel grids ( $200 \mathrm{mesh})$ and air-dried overnight. Autoradiograms prepared by both methods were incubated for $14 \mathrm{~d}$ at $4{ }^{\circ} \mathrm{C}$ and developed in $p$-phenylenediamine developer (Caro \& van Tubergen, 1962) for $1-2 \mathrm{~min}$ at $24^{\circ} \mathrm{C}$, washed in $2 \%(\mathrm{w} / \mathrm{v})$ acetic acid $(30 \mathrm{~s})$, fixed in $25 \%(\mathrm{w} / \mathrm{v})$ sodium thiosulphate $(5 \mathrm{~min})$ and rinsed in distilled water $(30 \mathrm{~min})$. Specimens were observed in a Siemens 102 transmission electron microscope (TEM) operated at $80 \mathrm{keV}$. Micrographs taken at magnifications up to $\times 80000$ were analysed by counting the number of silver grains over hyphal tip areas at $0.5 \mu \mathrm{m}$ intervals in the apical $4 \mu \mathrm{m}$.

Scanning electron microscopy of hyphal tips. Membrane-grown cultures were prepared by inoculation of MM agar plates overlaid by Cellophane membranes, prepared as described by Allan \& Prosser (1983), with either spore or mycelial suspensions. Samples of colonized membranes $(10 \mathrm{~mm} \times 5 \mathrm{~mm}$ in size) bearing mycelia from extreme marginal regions were excised and secured to aluminium plain-faced stubs using Aquadag colloidal graphite (Agar Aids). Gold-coated specimens were observed up to a magnification of $\times 10000$ using lowtemperature scanning electron microscopy (LTSEM) in a Cambridge S4 SEM interfaced to a Hexland cryo-system (EM Technology).

Quantitative morphometry of hyphal tips. Micrograph negatives from LTSEM preparations were enlarged and the shapes of 121 hyphal tips were analysed quantitatively by two methods. The first assumed tips to have circular transverse cross-section and semi-ellipsoid longitudinal cross-section defined by the lengths of semi-major and semi-minor axes. The length of the semi-major axis $(b)$ was calculated as the length of the line drawn from the hyphal apex parallel to the longitudinal axis which intercepted the semi-minor axis at right angles. The length of the semi-minor axis (a) was the length of the line drawn at right angles to 
the longitudinal axis at the point at which hyphal radius became constant. An ellipse is described by the equation:

$$
\frac{x^{2}}{a^{2}}+\frac{y^{2}}{b^{2}}=1
$$

The gradient of the ellipse at a point $P\left(x_{1}, y_{1}\right)$ is given by:

$$
\left(\frac{\mathrm{d} y}{\mathrm{~d} x}\right)_{i=\mathrm{a}}=-\frac{b^{2} x_{1}}{a^{2} y_{1}}
$$

The second method involved fitting polynomials to data points obtained by digitization of LTSEM tip profiles using a graphics tablet (Grafpad II, Hegotron Robotics). Data values were fitted to $n$ independent non-homogeneous equations in $n$ real unknowns by a least-squares method, which were then resolved by Gaussian elimination. This was done using programs run on a BBC model B microcomputer.

Treatment of mycelia with lysozyme and B-lactam antibiotics. A $10 \mathrm{ml}$ sample of an overnight culture of $S$. coelicolor in MEM was centrifuged at 1000 r.p.m. for $2 \mathrm{~min}$ and suspended in fresh MEM. Portions of this suspension were then treated with final concentrations of $100,200,300$ and $350 \mu \mathrm{g} \mathrm{ml}^{-1}$ of the following antibiotics: phenethicillin (potassium salt), methicillin, flucloxacillin, cloxacillin, carbenicillin (sodium salts), talampicillin hydrochloride, ticarcillin (sodium salt), amoxycillin (donated by Beecham Pharmaceuticals, Surrey, UK), cephalexin, penicillin $\mathrm{V}$ (potassium salt), cephaloridine, penicillin $\mathrm{G}$ (sodium salts) (donated by Glaxo Laboratories, Middlesex, UK) and ampicillin (Sigma). All were dissolved in buffered MEM except for talampicillin, for which ethanol $(50 \%, v / v)$ was required for dissolution. Control cultures were prepared by addition of solvent and medium only and all antibiotics were also added in the presence of $20 \%(\mathrm{v} / \mathrm{v})$ sucrose as an osmotic stabilizer. In addition, cultures were treated with filtersterilized hen egg-white lysozyme (Sigma) at final concentrations of $0 \cdot 01,0 \cdot 1,1,2,3,4,5,10,100$ and $1000 \mu \mathrm{g} \mathrm{ml}^{-1}$. Samples were incubated at $25^{\circ} \mathrm{C}$ and examined by phase-contrast microscopy at 2,3 and $4 \mathrm{~h}$, and 4 days. Samples of lysozyme-treated cultures were fixed $(2 \mathrm{~h})$ in paraformaldehyde $(2 \%, \mathrm{w} / \mathrm{v})$ and glutaraldehyde $(2 \cdot 2 \% \mathrm{v} / \mathrm{v})$ buffered (pH 7.2) with $200 \mathrm{~mm}$-sodium phosphate (Glauert, 1974), and then fixed $(0.5 \mathrm{~h})$ in osmium tetroxide $(1 \%, \mathrm{w} / \mathrm{v})$, dehydrated in an alcohol series and either critical-point-dried or added to coverslips pretreated with poly-L-lysine $(0.01 \%, w / v)$. Samples were sputter-coated with platinum, and observed using a Jeol 35 CF SEM operated at $10 \mathrm{keV}$ The viability of hyphae was determined by light microscopy following treatment with 3-(4,5-dimethylthiazol-2-yl)-2,5-diphenyltetrazolium bromide (MTT).

\section{Results}

\section{Incorporation of GlcNAc by hyphal tips}

Light microscopic autoradiography showed tritiated GlcNAc to be incorporated preferentially at hyphal tips and, to a lesser extent, at apices of lateral branches (Fig. $1 a)$. Quantitative analysis of the distribution of silver grains in sequential $5 \mu \mathrm{m}$ intervals showed the highest proportion of silver grains within the apical $5 \mu \mathrm{m}$ (Fig. $2 a$ ). Labelling in proximal regions was not due to residual unextracted GlcNAc or non-specific cytoplasmic labelling but suggested a small degree of wall synthesis, turnover or thickening. In pulse-chase cul-
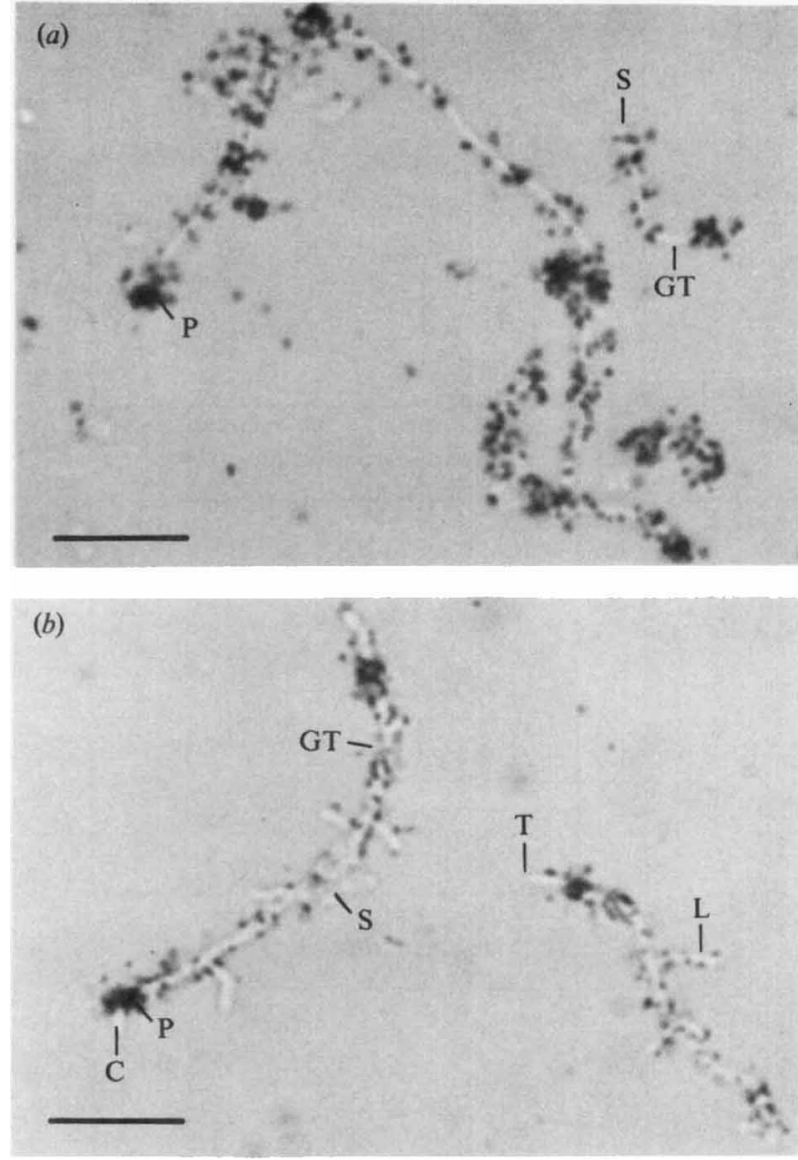

Fig. 1. Light microscopic autoradiograms of cultures of $S$. coelicolor labelled with tritiated GlcNAc. (a) A 3 min pulse-labelled culture of germinating spores grown as a submerged slide culture in MEM. Silver grains are visible as dark spots under bright-field conditions and are clearly seen to be located at the apices of hyphae. (b) A pulse-chaselabelled culture grown as in $(a)$ and then in the presence of $10 \mathrm{mg}$ nonradioactive GlcNAc ml-1 for $30 \mathrm{~min}$. Tips of lateral branches formed during the chase period are unlabelled, but the radioactivity in those tips present and elongating during the pulse labelling period show a dark region of silver grains distally located. T, hyphal tip; GT, germ tube: L, lateral branch; S, spore; P, pulse; $C$, chase. Bars, $10 \mu \mathrm{m}$.

tures, the apex was largely devoid of labelling, which was located in proximal regions (Fig. $1 b$ ). Two-step pulselabelling produced hyphae densely labelled in areas corresponding to the first labelling period and less densely in areas corresponding to the second period. Analysis of autoradiograms indicated a mean extension rate of $8.03 \mu \mathrm{m} \mathrm{h}^{-1}$ (SE $\left.0 \cdot 13, n=301\right)$.

High-resolution TEM autoradiograms also demonstrated apical incorporation of labelled wall precursors (results not shown). Quantitative analysis again showed a gradient of incorporation with distance from the tip (Fig. $2 b, c$ ). More than $50 \%$ of silver grains were found in the apical $0.5 \mu \mathrm{m}$ after incubation in the presence of label 

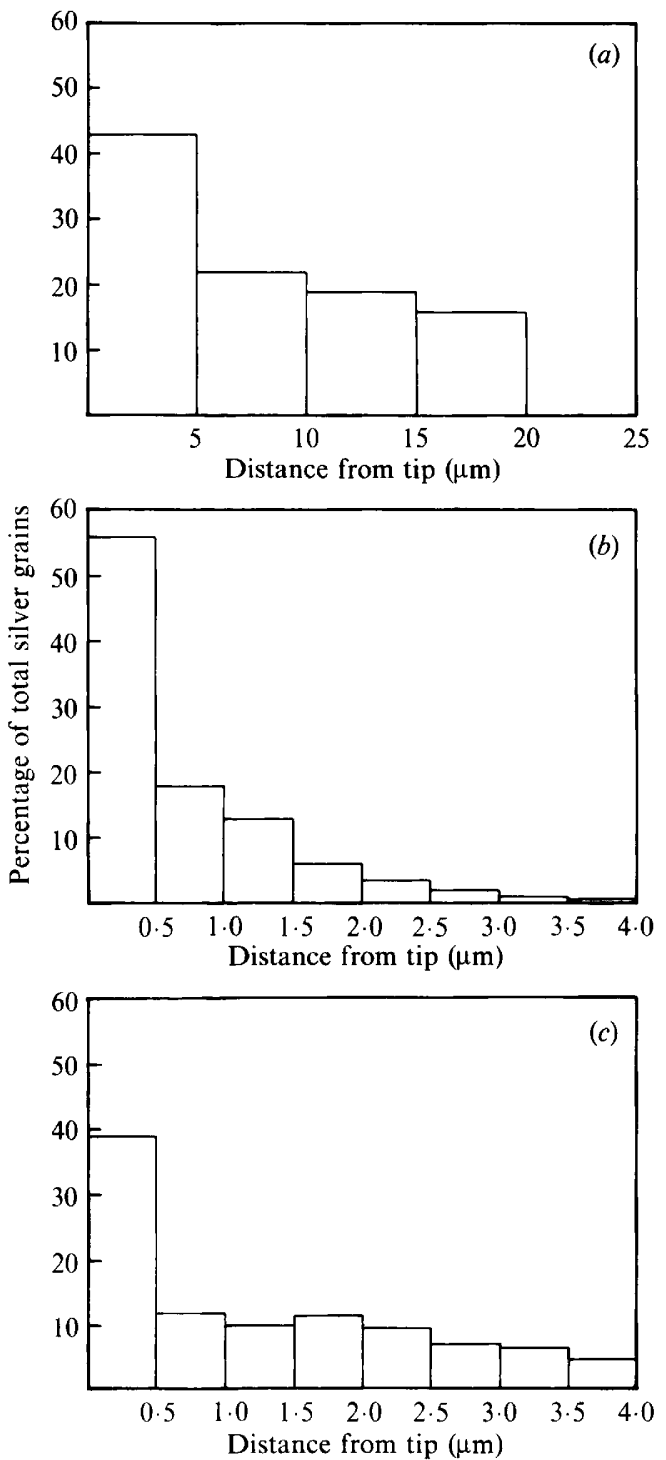

Fig. 2. Distribution of silver grains along hyphae of $S$. coelicolor labelled with tritiated GlcNAc. (a) Germ tubes were grown using the submerged slide culture technique, labelled for $3 \mathrm{~min}$ with tritiated GlcNAc and processed for light microscopic autoradiography. Autoradiograms were analysed using video-microscopy under darkfield conditions to count silver grains within $5 \mu \mathrm{m}$ intervals from the tips, expressed as a percentage of the total number of silver grains counted over each of the different distances measured from the apex. $(b, c)$ Young germlings were grown in liquid culture and labelled for $1 \mathrm{~min}(b)$, or $5 \mathrm{~min}(c)$, with tritiated Glc NAc at a final concentration of $1 \mu \mathrm{Ci} \mathrm{ml}^{-1}$. Labelled preparations were processed by high-resolution autoradiography using the flat substrate or the loop method with Ilford L. 4 emulsion. After $14 \mathrm{~d}$ the autoradiograms were observed by electron microscopy: silver grains were counted within successive $0.5 \mu \mathrm{m}$ intervals from the tips and were expressed as a percentage of the number of silver grains counted for each of the different pulse-labelling intervals.

for $1 \mathrm{~min}$. As the labelling period increased, this proportion fell below $40 \%$ through turnover of labelled material.

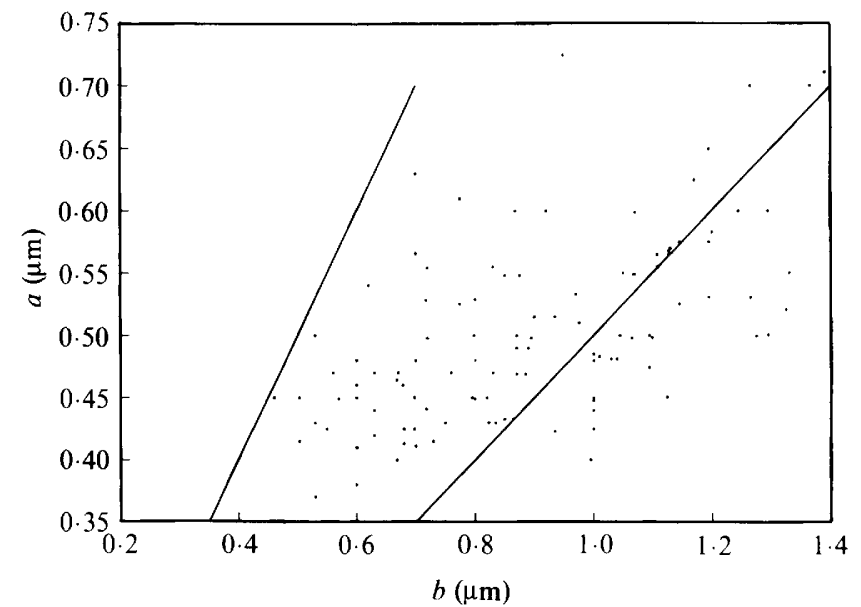

Fig. 3. A scatter diagram of hyphal eccentricity. The semi-major (b) and semi-minor $(a)$ axial distances have been plotted against each other for 121 hyphal tips. Hypothetical lines representing hemispherical (lefthand, $b / a=1$ ), or more ellipsoidal (right-hand, $b / a=2$ ) have been drawn.

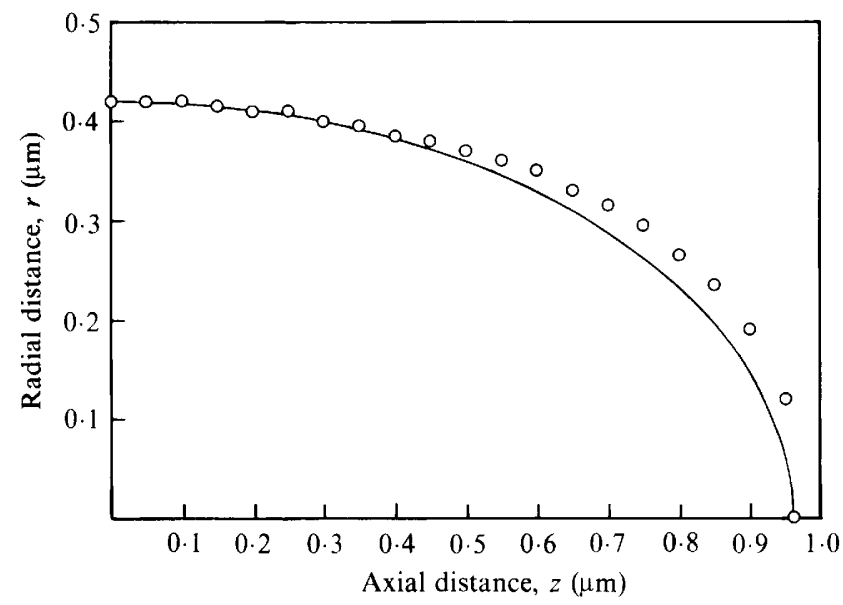

Fig. 4. Fitting of an ellipse to hyphal tip profile obtained from LTSEM micrographs. The profiles were digitized and an ellipse with semiminor axis equal to $z$ and semi-major axis equal to $r$ was fitted.

\section{Determination of hyphal tip shape}

Enlarged tracings of LTSEM micrographs were used to measure the lengths of semi-major $(b)$ and semi-minor $(a)$ axes of 121 hyphal tips (Fig. 3). A hemispherical tip will have $a=b$, but the data indicated values of $b / a$ approaching 2, implying shapes which are approximately semi-ellipsoid with a small degree of eccentricity. Fig. 4 shows a hyphal tip fitted to a semi-ellipse. The values of $a$ and $b$ were chosen to minimize the sum of squares of actual and predicted radial distances along the hyphal tip. Although approximating to semi-elliptical shape, tips tended to be flattened at the apex and polynomial equations were used to describe tip shape more accurately. 


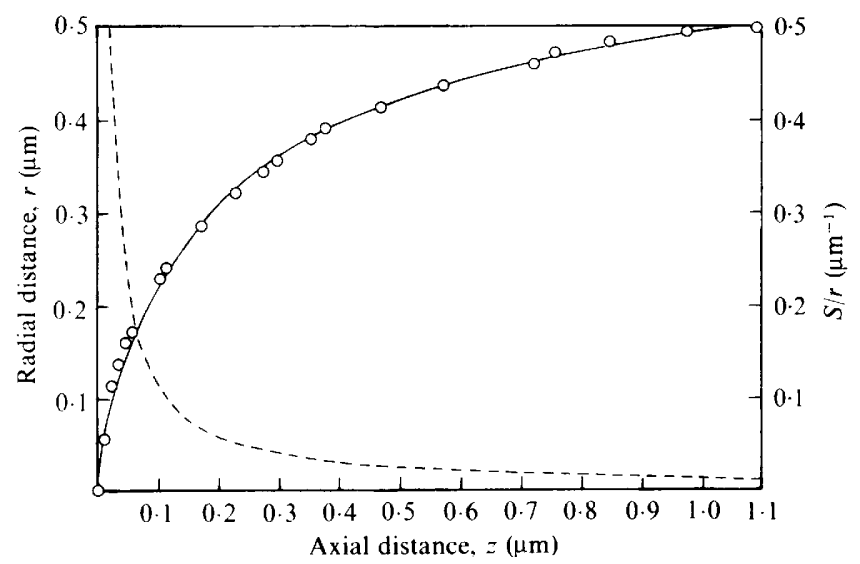

Fig. 5. Fitting of a polynomial to a hyphal tip profile obtained from LTSEM micrographs. Digitized points $(O)$ obtained from a tracing of a hyphal tip profile were used to determine the best-fitting polynomial. The hyphal tip profile illustrated had an axial distance of approximately $1 \mu \mathrm{m}$ and was fitted with a polynomial of degree 6 given by the function $\mathrm{f}(r)=65.9 r^{6}-9.44 r^{4}+2.41 r^{2}$. This equation was used to plot the graphs of hyphal tip shape (-) and the theoretical plot $(S / r$ plotted as a function of axial distance) over the tip profile (---).

The procedure was to calculate coefficients of polynomials by Gaussian elimination leading to a powerseries form of the polynomial which could be more readily manipulated. Equations were fitted to data obtained by digitizing enlarged tracings of hyphal tips from LTSEM micrographs. Most tips analysed could be represented well by polynomials of degree less than 7 and Fig. 5 illustrates the quality of fit of a tip to the equation:

$$
f(r)=65.9 r^{6}-9.44 r^{4}+2.41 r^{2}
$$

where $r$ represents radial distance. Equations of this type were then used to determine the tangent $(S)$, at a series of points on the hyphal tip. $S$ is equivalent to $\mathrm{d} r / \mathrm{d} z$, where $r$ represents radial distance, and its variation with axial distance $(z)$ defines tip shape. The quantity $S / r$ is inversely related to surface tension $(T)$ within the wall (Koch, 1982) and thereby reflects apparent wall elasticity. Variation in $S / r$ with distance from the hyphal apex decreases markedly within $25 \%$ of the extension zone length (Fig. 5). Wall elasticity cannot be measured directly but is considered to be related to the specific rate of wall growth, here measured as the rate of incorporation of labelled GlcNAc. Comparison of variation in $S / r$ (Fig. 5) and gradients in the rate of incorporation (Fig. $2 b, c)$ demonstrate good qualitative agreement.

\section{Alteration of hyphal tip morphology}

Concentrations of lysozyme greater than $0.1 \mu \mathrm{g} \mathrm{ml}^{-1}$ resulted in lysis of germ tubes produced from spores in liquid medium. Treatment of 1-d-old submerged cultures with a lysozyme concentration of $1 \mu \mathrm{g} \mathrm{ml} \mathrm{m}^{-1}$ also produced widespread lysis while in the presence of $20 \%$ $(\mathrm{w} / \mathrm{v})$ sucrose, lysis was prevented but swellings were observed at hyphal apices. Following removal of lysozyme some of the tip swellings developed more pointed apices, implying a resumption of normal hyphal growth.

Application of $5 \mu \mathrm{g}$ lysozyme $\mathrm{ml}^{-1}$ to hyphae at the margins of membrane surface cultures, on solid medium, resulted in apical lysis and production of transverse corrugations of cell-wall material. At lower concentrations, lysozyme produced a range of morphologies. Fig. 6 illustrates hyphae of cultures grown in liquid medium in the absence of lysozyme for $12 \mathrm{~h}$ and subsequently for $12 \mathrm{~h}$ in the presence of $1 \mu \mathrm{g}$ lysozyme $\mathrm{ml}^{-1}$ (final concentration). Hyphal swelling was commonly observed, frequently without any apparent preferential site (Fig. $6 a, c, d$ ) and all areas appeared roughened in critical-point-dried SEM preparations. The swellings often gave a beaded appearance. It was not possible to determine whether this resulted from a simultaneous swelling along the whole length of a hypha or from continued extension of a swollen tip. The beaded appearance may be associated with formation of septa, which is also indicated by the appearance in Fig. 6(a,c) of a lysed region bounded by two swollen hyphal regions. In Fig. 6(b), normal hyphae appear to have developed from a swollen region, although this morphology may have resulted from swellings in proximal regions of the hyphae where cell walls may be more susceptible to lysozyme attack.

Mycelia cultured in liquid medium showed a range of sensitivities to the different $\beta$-lactam antibiotics. At $100 \mu \mathrm{g} \mathrm{ml}^{-1}$, they were most sensitive to ampicillin and cephaloridine, showing hyphal lysis after $2 \mathrm{~h}$ with no recovery. Cephalexin, penicillins $\mathrm{G}$ and $\mathrm{V}$, talampicillin and amoxycillin gave lysis or distorted hyphae, but required higher concentrations to prevent recovery to normal growth after $4 \mathrm{~d}$. Methicillin and flucloxacillin required $200 \mu \mathrm{g} \mathrm{ml}^{-1}$, carbenicillin and ticarcillin required $300 \mu \mathrm{g} \mathrm{ml}^{-1}$ and cloxacillin required $350 \mu \mathrm{g} \mathrm{ml}^{-1}$ before lysis was seen, but all mycelia recovered after $4 \mathrm{~d}$. Phenethicillin had no discernible lytic effect.

When mycelia were grown as surface membrane cultures, however, a different pattern of effects was seen after treatment with the antibiotics in the presence of $20 \%(\mathrm{w} / \mathrm{v})$ sucrose. Lysis was prevented, except after prolonged incubation with cephaloridine $(42 \mathrm{~h})$, penicillin $G$ and talampicillin $(83 \mathrm{~h})$. In all cases except methicillin, the treatments resulted in rounded or elongated apical swellings, first with cephaloridine, penicillins $G$ and $V$, ampicillin and cephalexin (by $155 \mathrm{~min}$ ), then with cloxacillin and carbenicillin (by $180 \mathrm{~min}$ ), then with talampicillin, flucloxacillin, ticarcil- 

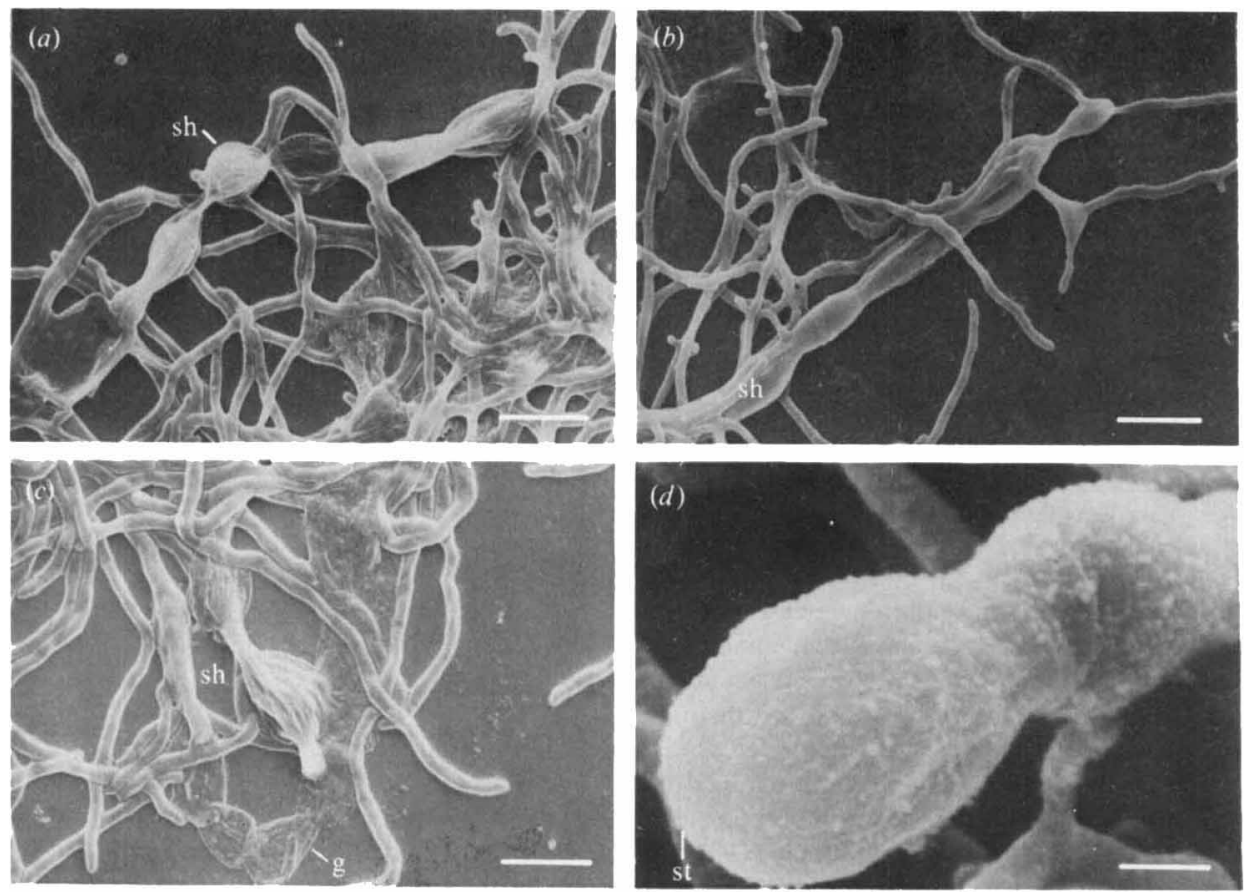

Fig. 6. SEM micrographs showing the effects of lysozyme on hyphal morphology. Hyphae were grown from spores in $2 \%$ (w/v) MEM for $12 \mathrm{~h}$, then transferred to the same medium but containing $1 \mu \mathrm{g}$ hen egg-white lysozyme $\mathrm{ml}^{-1}$. Following a further $12 \mathrm{~h}$ of growth, samples were removed for SEM examination. Preparations seen in $(a)$ and $(c)$ were air-dried whole, all others were prepared by criticalpoint-drying of specimens. (a) Swollen hyphae which have collapsed due to air drying. In the centre of the micrograph a lysed portion is bounded by two 'swollen' ones thus indicating that beading is due to septa. (b) The degree and extent of hyphal swelling (as observed in critical-point-dried preparations) is easily seen in this micrograph. Normal hyphae appear to originate from two swollen hyphae (sh). (c) A cell-wall ghost (g), which maintains the shape of the stretched hypha. (d) A swollen hyphal tip (st) preserved by critical-point-drying. This tip is approximately $2.5 \mu \mathrm{m}$ in diameter and its surface appears roughened due to the lytic action of lysozome. Bars: $5 \mu \mathrm{m}(a, b, c)$, $1 \mu \mathrm{m}(d)$.

lin and amoxycillin (by $290 \mathrm{~min}$ ) and then with phenethicillin (by $700 \mathrm{~min}$ ). Protoplasts were formed in all cases except treatment with methicillin and flucloxacillin. They were seen earliest with cephaloridine, cloxacillin, carbenicillin and cephalexin (by $180 \mathrm{~min}$ ), then with penicillins $\mathrm{G}$ and $\mathrm{V}$, ticarcillin, amoxycillin, ampicillin and talampicillin (by $290 \mathrm{~min}$ ) and then with phenethicillin (by $700 \mathrm{~min}$ ).

\section{Discussion}

The data presented here provide further evidence for apical growth of actinomycete hyphae. Light microscopic autoradiography demonstrated apical incorporation of labelled GlcNAc, a precursor of peptidoglycan, and pulse-chase experiments confirmed this finding. Reduced incorporation by apices of lateral branches may reflect their lower diameter and extension rate. The hyphal extension rate calculated from autoradiographs of cultures growing in liquid MEM $\left(8 \mu \mathrm{m} \mathrm{h}^{-1}\right)$, is less than that reported for growth of this organism on solid medium (10-13 $\mu \mathrm{m} \mathrm{h}^{-1}$; Allan \& Prosser, 1983). This may be due to differences in growth media or to alteration of the balance between extension rate and branching density under the different growth conditions.

There was some evidence for incorporation of label behind the tip but there was no pattern to the distribution of this material and it probably resulted from metabolism of GlcNAc, turnover of wall material or wall thickening. Miguelez et al. (1988) suggested a multisite zonal model for growth of hyphae of $S$. antibioticus, immediately following spore germination. They reported stepwise increases in extension rate as the number of growth zones increased, but labelling patterns predicted by this mode of growth were not observed in S. coelicolor. This may be due to our studying hyphae in older cultures with a constant extension rate but Kretschmer (1988), who observed similar stepwise increases in extension rate of Streptomyces granaticolor, suggested that these might arise from incremental increases in the synthesis and supply of material to the hyphal apex, rather than multiple growth zones.

Electron microscopic autoradiography increased the resolution of labelling studies and enabled quantification of labelling patterns within the extension zone. This provided further evidence for apical growth with $57 \%$, 
$75 \%$ and $87 \%$ of label incorporated in the apical $0.5 \mu \mathrm{m}$, $1.0 \mu \mathrm{m}$ and $1.5 \mu \mathrm{m}$ respectively after labelling for $1 \mathrm{~min}$. The significant redistribution of label to more distal regions following longer pulses of labelling indicates rapid metabolism and turnover of GlcNAc.

Localized swelling of hyphae in apical regions following treatment with $\beta$-lactam antibiotics also indicates hyphal extension by apical growth. These antibiotics specifically inhibit the final stages of peptidoglycan synthesis, without affecting cellular growth. Apical swelling presumably resulted from an increase in internal hydrostatic pressure acting on the tip wall whose rigidification was prevented. $\beta$-Lactam antibiotics will only affect regions of active hyphal extension and the beaded morphology which would be predicted by multisite zonal models was not observed. Lysozyme, however, cleaves alternate $\beta(1-4)$ linkages between GlcNAc and $N$-acetylmuramic acid residues and acts on preformed peptidoglycan. Treatment with lysozyme gave rise to a greater range of morphological alterations, with swelling at hyphal apices and in more distal regions. This presumably resulted from internal hydrostatic pressure acting on wall material softened by enzymic degradation. The beaded morphology frequently extended some distance behind the tip and appeared to result from the presence of septa, with the septal wall protected from lysozyme activity.

Apical hyphal swelling and the relationship between GlcNAc incorporation and tip shape provide strong support for the application of the surface stress theory to the apical extension of streptomycete hyphae. Tip growth is therefore driven by internal hydrostatic pressure and the tip assumes a shape which minimizes surface energy and reflects variation in surface tension within the wall, which in turn reflects variation in the specific rate of wall growth and wall rigidification. For fungal hyphae, there is now strong evidence (Wessels, 1986) that new wall material is incorporated at the hyphal apex in plastic form and then rigidifies in a timedependent process through covalent cross-linking of wall components. A similar process may occur in prokaryotes (de Pedro \& Schwarz, 1981). In Streptococcus faecalis (Dezelee \& Shockman, 1975) and Bacillus megaterium (Fordham \& Gilvarg, 1974) there appear to be two phases of peptidoglycan incorporation. The first involves rapid incorporation of soluble peptidoglycan into insoluble material which is followed by a 'remodelling' phase. Evidence for a similar process in streptomycetes comes from the existence of two types of transpeptidase in Streptomyces R61 (Leyh-Bouille et al., 1977; Dusart et al., 1977). The first is strongly associated with the cell membrane, begins cross-linking of nascent peptidoglycan strands and is relatively insensitive to $\beta$-lactam antibiotics. The second is weakly associated with the membrane and sensitive to $\beta$-lactam antibiotics. Inhibition of this second phase, in the presence of an osmotic stabilizer, could have given rise to spherical swelling and limited subsequent growth may have led to the elongated swellings which were sometimes observed.

Hyphal tip shape could be approximated by a semiellipse, of circular cross-section, but the eccentricity of the observed shapes was much less than those found in fungal hyphal tips (Trinci \& Saunders, 1977). The rate of rigidification will determine hyphal diameter but the eccentricity of tip shape will depend on the pressure difference across the tip wall and the steepness of the gradient in surface tension within the extension zone. These differences may therefore reflect differences between streptomycetes and fungi in internal hydrostatic pressure or, more probably, differences in the detailed mechanism of incorporation of new wall material. It is presumed that material is transported to hyphal tips of streptomycetes by diffusion, while fungal hyphae possess complex transport systems involving membrane-bound vesicles and cytoskeletal structures. This may alter the way in which material is directed to points within the extension zone or may merely reflect the smaller size of streptomycete hyphae.

We thank Drs D. Gregory and W. J. McHardy and Mr J. M. Tait for assistance with electron microscopy. This work was supported by the Science and Engineering Research Council Research Grant GR/C91826.

\section{References}

Allan, E. J. \& Prosser, J. I. (1983). Mycelial growth and branching of Streptomyces coelicolor A3(2) on solid medium. Journal of General Microbiology 129, 2029-2036.

Allan, E. J. \& Prosser, J. I. (1985). A kinetic study of the colony growth of Streptomyces coelicolor A3(2) and J802 on solid medium. Journal of General Microbiology 131, 2521-2532.

Brana, A. F., Manzanal, M.-B.\& Hardisson, C. (1982). Mode of cell wall growth of Streptomyces antibioticus. FEMS Microbiology Letters 13, 231-235.

Caro, L. G. \& van Tubergen, R. P. (1962). High resolution autoradiography. I. Methods. Journal of Cell Biology 15, 173-188.

Dezelee, P. \& Shockman, G. (1975). Studies of the formation of peptide cross-links in the cell wall peptidoglycan of Streptococcus faecalis. Journal of Biological Chemistry 250, 6806-6816.

Dusart, J., Leyh-Bouille, M. \& Ghuysen, J.-M. (1977). The peptidoglycan cross-linking enzyme system in Streptomyces strains R61, K 15 and rimosus. Kinetic coefficients involved in interactions of the membrane-bound transpeptidase with peptide substrates and $\beta$-lactam antibiotics. European Journal of Biochemistry 81, 33-44.

Fordham, W. D. \& Gilvarg, C. (1974). Kinetics of cross-linking of peptidoglycan in Bacillus megaterium. Journal of Biological Chemistry 249, 2478-2482.

Glauert, A. M. (1974). Practical Methods in Electron Microscopy 3 Fixation, Dehydration and Embedding of Biological Specimens. Amsterdam: North Holland Publishing Co.

Gooday, G. W. \& TRINCI, A. P. J. (1980). Wall structure and biosynthesis in fungi. In The Eukaryote Microbial Cell, pp. 207-251. Edited by G. W. Gooday, D. Lloyd \& A. P. J. Trinci. London: Cambridge University Press. 
GrEen, P. B. (1974). Morphogenesis of the cell-organ axis biophysical models. Brookhaven Symposia in Biology 25, 166-190.

Hopwood, D. A. (1960). Phase contrast observations on Streptomyces coelicolor. Journal of General Microbiology 22, 295-302.

$\mathrm{KoCH}, \mathrm{A}$. L. (1982). The shape of the hyphal tips of fungi. Journal of General Microbiology 128, 947-951.

Koch, A. L. (1983). The surface stress theory of microbial morphogenesis. Advances in Microbial Physiology 24, 301-366.

KoCH, A. L. (1985). How bacteria grow and divide in spite of internal hydrostatic pressure. Canadian Journal of Microbiology 31, 10711084 .

KRETSCHMER, S. (1988). Stepwise increase of elongation rate in individual hyphae of Streptomyces granaticolor during outgrowth. Journal of Basic Microbiology 28, 35-43.

Leyh-Bouille, M., Dusart, J., Nguyen-Disteche, M., Ghuysen, J.-M., Reynolds, P. E. \& Perkins, H. R. (1977). The peptidoglycan cross-linking enzyme system in Streptomyces strains R61, K15 and rimosus. Exocellular, lysozyme-releasable and membrane-bound enzymes. European Journal of Biochemistry 81, 19-28.
Locci, R. \& SchaAl, K. P. (1980). Apical growth in facultative anaerobic actinomycetes as determined by immunofluorescent labelling. Zentralblatt für Bakteriologie 246, 112-118.

Miguelez, E. M., Martin, M. C., Manzanal, M. B. \& Hardisson, C. (1988). Hyphal growth in Streptomyces. In Biology of Actinomyces' 88 , pp. $490-495$. Edited by Y. Okami, T. Beppi \& H. Ogawara. Tokyo: Japan Scientific Societies Press.

de Pedro, M. A. \& Schwarz, U. (1981). Heterogeneity of newly inserted and pre-existing murein in the sacculus of Escherichia coli. Proceedings of the National Academy of Sciences of the United States of America 78, 5856-5860.

Rogers, A. W. (1973). Techniques of Autoradiography, 2nd edn. Amsterdam: Elsevier.

SaUnders, P. T. \& TRINCI, A. P. J. (1979). Determination of tip shape in fungal hyphae. Journal of General Microbiology 110, 469-473.

Trinci, A. P. J. \& SAunders, P. T. (1977). Tip growth of fungal hyphae. Journal of General Microbiology 103, 243-248.

WESSELS, J. G. H. (1986). Cell wall synthesis in apical hyphal growth. International Reviews of Cytology 104, 37-79 